\title{
Displasia Broncopulmonar Grave
}

\author{
Dr. Juan Pable Bcca I.' Dr. Jolue Wilson Sch.; Dr. Lionel Bermuci V.: \\ De. Jorge Torses P.
}

\section{Bronchopulmonary Dysplasia}

\begin{abstract}
Seyere Bronchopulmonary dysplasia (BPi); wecurring in 4 babius with gestational ages from 24 to 28 weeks and birthweights from 700 to 1.170 gr. In described. All of theme were manged with 1PPV with moderate settings lor 41

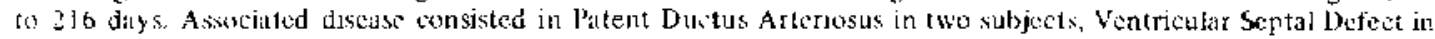
one. Cy tomegalowirus infestion in one. Severio larylgea obstenction occurred in two cases. Oxygen therapy had to

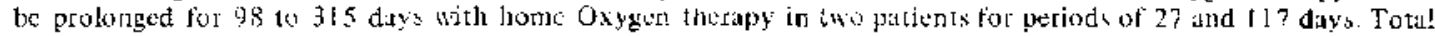
hospital stays runged from 100 to 273 day's. All lou inlants were neurolugically nermal at tollow up at adjuster ilges ot 8 to 24 monthe but one of them in still severely growth retarded. Prognosis of BPD appears tis be

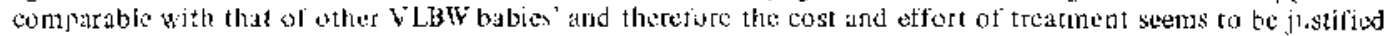
(Key word:: Bronchopulmonary dysplussá. Mechanical ventilation. P'rematurity).
\end{abstract}

La Displasia Broncopulmonar (Dbl') es una enfermedad pulmontar crónica del prematuro caracterizada por dano del epitelio alveolar $y$ bronquial seguido de metaplasia bronquiolar $y$ fibrosis peribronquial e intersticial. se relaciona con el uso de ventiladores mecánicos y se manifiesta clínicamente por persistencia de la difïcultad respiratoria. dependencia prolongada de uxígeno adicional $y$ alteraciones radiológicas características ${ }^{2}$.

A medida que sobreviven más recien nacidos

1. Servicio de Neonatologia, Clínica Alemana, Santiago. de bajo peso tratados con ventilación mecánica, como consecuencia del desarrollo experimentado en los últimos años en el país por las técnicas de cuidado intensivo neonatal, era de esperar la aparición de nuevos casos de DBP severa.

Nuestro grupo ha seguido cuatro pacientes con DBP a lo largo de 1 a 2.5 años. Comunicamos los resultados del seguimiento y los hechos clinicos y de laboratorio mas notables de estos pacientes.

\section{PACIENTES Y METODOS}

Se revisaron retrospectivamente los anteceden. tes de cuatro pacientes con Displasia Broncopulmonar (DBP). 
Se definió como DBP una enfermedad pulmonar crónica que ocurre en pacientes nacidos prematuramente, relacionada con el empleo de ventilación mecánica por tiempos prolongados. caracterizada por persistencia del síndrome de dificultad respiratoria que originó la indicación de ventilación mecánica más allá de $\operatorname{los} 7$ a 10 días de vida, dependencia de suministro adicional de oxigeno por más de 30 días y presencia de las alteraciones radiologicas que Northway ${ }^{2-5-7}$ ha dividido en cuatro etapas: cambios inespecificos indistinguibles del patrón caracteristico del sindrome de membrana hialina (Etapa 1); opacificación casi total del pulmón con persistencia del broncograma (Etapa 2); infiltrados de aspecto quístico (Etapa 3); hipertransparencia e infiltrados en cordón que representan fibrosis bronquiolar e intersticial (Etapa 4):

Todos los pacientes nacieron en la Clínica Alemana de Santiago, eran prematuros extremos, debieron ser conectados a un ventilador mecánico antes de las 6 horas de vida y fueron manejados de acuerdo con los procedimientos estándar para estos casos en una unidad de tratamiento intensivo neonatal (UTIN).

El tratamiento empleado consistió en ventilación mecánica con presión positiva intermitente (IPPV), drenaje de secreciones por gravedad y aspiración, antibióticos cuando se sospechó infección bacteriana.

\section{RESULTADOS}

Los hechos clínicos más destacados de estos recién nacidos están en la Tabla 1.
La edad gestacional varió entre 24 y 28 semanas, el peso de nacimiento entre $700 \mathrm{y}$ $1.170 \mathrm{gr}$.: 3 fiueron de sexo masculino; el parto fue espontáneo en tres casos y por cesárea en uno. Dos de los cuatro casos tuvieron Apgar al minuto menor de 3 .

La ventilación mecanica se indicó en todos antes de las 6 horas de vida, por Membrana Hialina (SDRI) $(n=2)$, Bronconeumonia o Apnea y se prolongó por períodos que variaron entre 41 y 216 días. Las variables ventilatorias empleadas consistieron en presiones inspiratorias máximas (PIM) entre 20 y $30 \mathrm{~cm}, \mathrm{H}_{2} \mathrm{O}$, presiones positivas al final de la expiración (PEEP) hasta $6 \mathrm{~cm}$. de $\mathrm{H}_{2} \mathrm{O}$ y presiones medias de la vía aérea (MAP) que resultaron siempre inferiores a $11 \mathrm{~cm} . \mathrm{H}_{2} \mathrm{O}$. La fracción inspiratoria de Oxígeno $\left(\mathrm{FiO}_{2}\right)$ se ajustó según los gases arteriales y la oximetria transcutänea, siendo los requerimientos de todos los pacientes de 0,50 o mayores por plazos de 3 a 81 días. Uno de ellos requirió $\mathrm{FiO}_{2}$ sobre 0,70 por 13 días. (Tabla 1 ).

Los intentos por retirar a los pacientes del respirador en la primera semana fracasaron por deterioro de las concentraciones de los gases arteriales o crisis de apnea y desde la segunda semana de vida pudieron reconocerse en ellos manifestaciunes radiológicas sugerentes de DBP.

La evolución clínica en todos los casos fue arrastrada, con hipoventilación, zonas atelectásicas de tamaño y duración variables y periodos de hipersecreción; dos de los casos sufrieron además obstrucción de la via aérea alta, que en uno (Caso 4) obligó a prolongar la ventilación mecánica. La dependencia de Oxígeno se mantuvo por plazos prolongados que variaron de 95 a

Tabla 1.

Antecedentes Clínicos de 4 Casos de Displasia Broncopulmonar Grave.

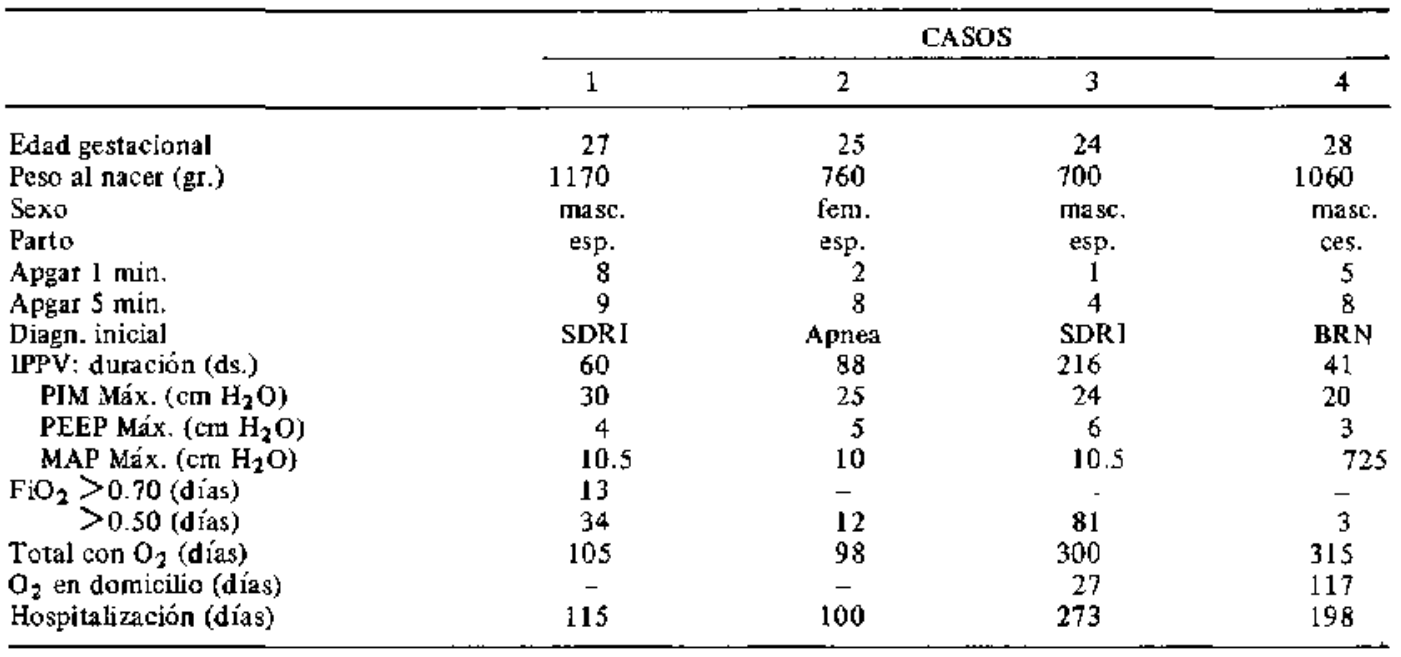


315 dias $y$ dos pacientes debieron continuar recibiéndolo en sus domicilios, después del alta.

Las radiografias mostraron inicialmente opacidad difusa inespecífica y en la segunda semana, imágenes específicas intersticiales difusas con hiperinsuflacion en ambos campos pulmonares (Figura 1). En las etapas avanzadas se observaron zonas hiperaireadas alternadas con otras de atelectasia (Figura 2). La normalización radiológica ocurrió en placos variables que alcanzaron hasta más de un año.

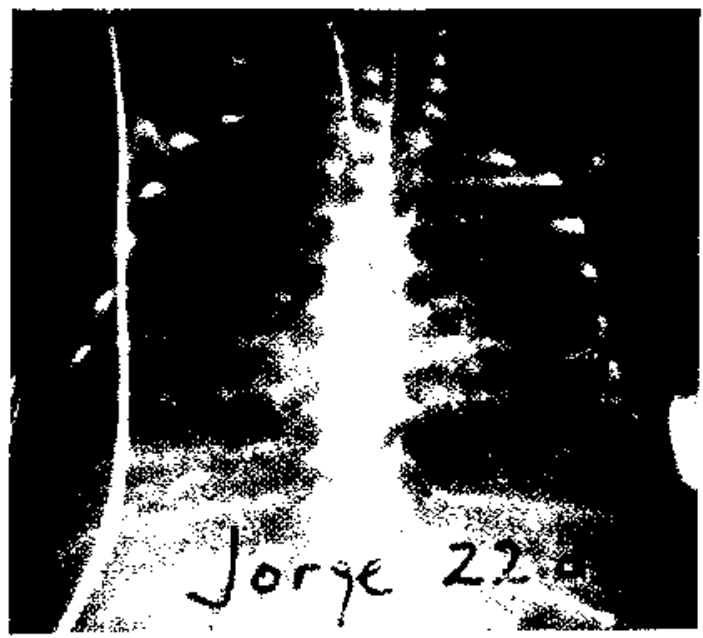

Figura 1: Radiografíli sugerente de DBP en R.N. de pretérmino de 22 días (caso 3) mostrando imágenes intersticiales difusas con hiperitsultacion en ambos campos pulmonates.

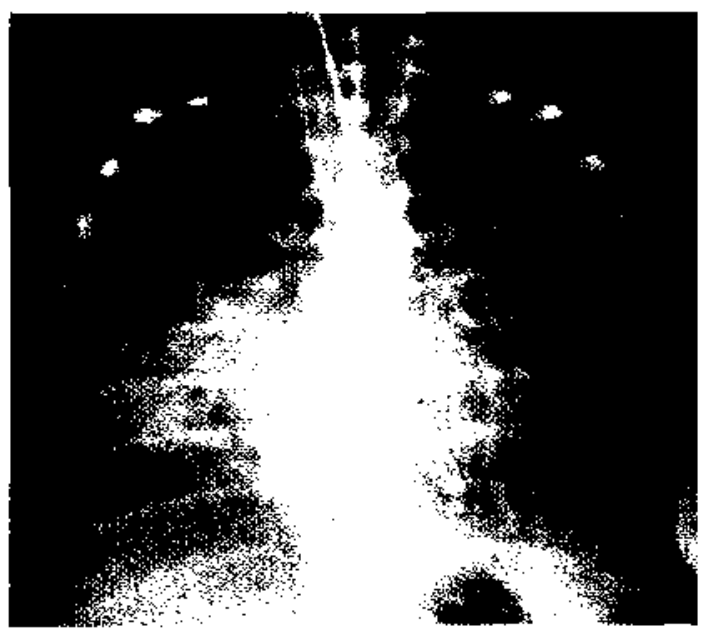

Figura 2: Radiografía tomada a los 40 días (Laso 3) mostrando zonas hiperaireadus aiternadas con zonas atelcctásicas en etapa avanzada de DBP.

En los dos primeros casos se usó Furosemida de manera ocasional y en los dos ríltimos en forma sistemática, en dosis de 1 a $3 \mathrm{mg} / \mathrm{Kg} / \mathrm{dia}$ por vía oral por 2 a 3 meses.
En los dos primeros casos se intentó, sin resultados positivos, cerrar el Ductus con Indo. metacina oral; el cierre ocurrió espontáneamente en ambos después de los tres meses de edad. En los otros dos niños no se encontró evidencia de permeabilidad del Ductus, pero uno tenía un soplo sistólico sugerente de comunicación interventricular que desapareció después del alta. Tres pacientes tuvieron complicaciones laringeas asociadas al procedimiento de ventilación: El Caso 1 presentó signos severos de obstrucción laríngea al extubarlo: que se resolvieron después de la inyección sub-glótica y la administración por vía intramuscular y local en aerosol de corticoides. El caso 3 también sufrió ubstrucción laríngea severa pero no respondió a los corticoides locales $y$ generales, obligando a prolongar la ventilación mecanica a pesar de la mejoría de la función pulmonar. En el último control, a los 14 meses de edad persistia en la laringoscopía una sinequia de la horquilla anterior de las cuerdas vocales que causaba estridor y retracción torácica permanentes. Un tercer caso (Caso 2) presentó disfonia que en menor grado ha persistido hasta -l último control a los 2 afios de edad. Ninguno de los casos tuvo evidencia clinica o ecoencefalografica de Hemorragia Intracraneana.

El caso 3 sufrió una infección por Citomegalovirus con púrpura trombocitopénico que fue demostrada por aislamiento del virus en la orina, lo que se atribuyó al uso de transfusiones para reponer su volumen sanguíneo. El mismo niño presenté signos radiológicos y bioquímicos de raquitismo con fracturas costales $y$ fractura patológica de cémut. El caso 2 presentó también fracturas costales y signos de raquitisno.

Los pacientes descritos han sido seguidos hasta edades cronológicas que Eluctúan entre 11 y 27 meses, que corresponden a edades corregidas entre 8 y 24 meses. Como se aprecia en la Tabla 2 sus pesos, tallas y circunferencias craneanas están en rangos normales para la edad corregida en tres de los cuatro. Sólo el Caso 3 tenia, a la edad corregida de 8 meses, un retardo pondo. estatural severo. En la Figura 3 se muestran las curvas de crecimiento correspondientes: Los parámetros, inicialınente bajos, se recuperaron en el tiempo acercándose a la normalidad salvo en uno de los casos

El desarrollo psicomotor ha sido normal en todos los casos, ninguno tiene signos de dafio o déficit neurológico o sensorial hasta la edad del seguimiento.

Dus de los casos debieron ser rehospitalizados. El caso 2 por 34 días debido a bronconeumoria $y$ atelectasia pulmonar grave un mes después del alta; posteriormente fue hospitalizada dos veces mas por 24 horas para realizar herniortafia in- 
Tabla 2.

Seguimiento de 4 Casos de Displasia Broncopulmonar Grave.

\begin{tabular}{|c|c|c|c|c|}
\hline & \multicolumn{4}{|c|}{ CASOS } \\
\hline & 1 & 2 & 3 & 4 \\
\hline Edad cronológica (meses) & 27 & 19 & $111 / 2$ & $111 / 2$ \\
\hline Edad corregida (meses) & 24 & $151 / 2$ & 8 & $8 \mathrm{l} / 2$ \\
\hline Peso (petcentil) & $25-50$ & $>90^{\circ}$ & $<3$ & 25 \\
\hline Talla (percentil) & 75 & 90 & $<10$ & $>50$ \\
\hline Circ. cráneo (precentil) & 50 & 50 & $<10$ & 50 \\
\hline Desarrollo psizomotor & normal & notmal & normal & normal \\
\hline Sensorial & normal & normal & normal & normal \\
\hline Hospitalizaciones (n) & - & 3 & - & 1 \\
\hline
\end{tabular}

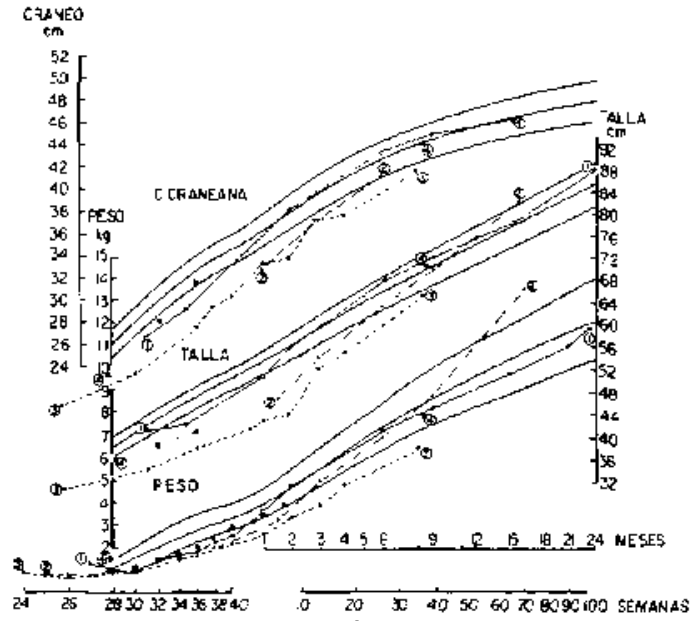

Figura 3: Curvas de crecimiento, peso y circunferencia craneana de 4 casos de DBP grave desde el nacimiento hasta edad corregida de seguimiento a $\operatorname{los} 8$ a 24 meses, de acuerdo a las Tablas de Gairdner y Pearson ${ }^{6}$.

guinal y tratar una infección urinaria respectivamente. El Caso 4 fue hospitalizado 7 dias por bronconeumonia sin caracteres de gravedad (Tabla 2).

\section{DISCUSION}

Dentro de estos criterios relativamente ant $^{-}$ plios para identificar DBP, los casos presentados corresponden a formas especialmente graves que permiten revisar los factores y el pronóstico a mediano plazo.

La incidencia de la DBP varia en distintas series entre 5 y $38 \%$ de los RN sometidos a vertilación mecánica con mortalidad entre 25 y $39 \%^{1 \cdot 7}$. Nuestra experiencia, sin mortalidad, corresponde a una serie muy pequeña y no permite comparaciones de sobrevida o incidencia.

Los factores etiopatogénicos de DBP citados en la literatura incluyen prematuridad extrema, uso prolongado de aitas concentraciones de Oxígeno, presiones positivas elevadas en respirador mecánico, intubación endotraqueal prolon. gada, persistencia del Ductus Arterioso, administración excesiva de líquidos, déficit de vitamina E e inflamación crónica $2 \cdot 3-4 \cdot 5 \cdot a \cdot 9 \cdot 10 \cdot 11$. 12 , todos difíciles de analizar por separado. Los pacientes de esta serie fueron prematuros extremos, necesitaron ventilación mecánica prolongada, si bien con presiones moderadas, y empleo prolongado, aunque relativamente restringido de Oxígeno.

En otras series las infecciones bacterianas intrahospitalarias pueden alcanzar mayor gravedad y frecuencia que la obseryada en esta experiencia. También, suele esperarse en este tipo de enfermos la aparición de Hemorragia Intracraneana, ausente en nuestra casuística.

E1 tratamiento de la DBP consiste fundamentalmente en compensar la insuficiencia respiratoria mediante ventilación mecánica cautelosa, uso de Oxígeno adicional según requerimientos, kinesioterapia respiratoria, antibióticos si se sospecha infección y aportes nutricionales adecuados, eventualmente con apoyo parenteral. Se ha propuesto también el uso de cortjcoides, con dudosos resultados ${ }^{3-14}$, de Furosemida y de Oxígeno con Helio (Heliox) para mejorar la mecánica pulmonar ${ }^{15}$. Resulta interesante destacar la experiencia positiva en los dos casos en que se uso Oxígeno en el domicilio: Se requiere capacitación de la familia, pero tiene grandes ventajas afectivas, económiças y de estimulación, como ha sido descrito en otras experiencias ${ }^{16}$.

Los RN con descarga diurética precoz en el curso del SDRI tienen menor incidencia de DBP17.18, y, el mayor aporte de líquidos se asocia a mayor incidencia de DBP1 1 por ello se ha recomendado el uso de Furosemida, pero no está claro que con ella se logre prevenir la DBP $^{19}$. La Furosemida además de su acción diurética ejerce un efecto de reducción de la obstrucción bronquial en niños con DBP, posi- 
blemente relacionado con la disminución del edema y por consiguiente de la resistencia de la vía aérea. El efecto diurético "crónico" sobre la evolución de la DBP, no es claro y tiene riesgos de hipercalciuria, raquitismo y calcifícaciones renales ${ }^{20}$ por lo que su empleo debe ser considerado cuidadosamente. Tenemos la impresión que la Furosemida podría tener una acción favo. rable en los períodos de mayor descompensación respiratoria y retención de $\mathrm{CO}_{2}$, en cambio es discutible el beneficio de su uso prolongado. Ambas afirmaciones requieren de estudios controlados en series mayores.

El Ductus puede jugar un rol en la etiopatogenia de la DBP por la congestión pulmonar que se produce secundariamente al cortocircuito de izquierda a derecha. Existe relación entre su persistencia y la DBP. Así, en recién nacidos con peso inferior a $1.500 \mathrm{gr}, \mathrm{y}$ Ductus persistente al tercer día, hay DBP en el $68 \%$ de los casos en contraste con $22 \%$ entre aquellos sin persistencia del Ductus ${ }^{8}$. El cierre precoz del Ductus disminuye la incidencia de $\mathrm{DBP}^{10-21}$, pero persiste la dificultad de efectuar el diagnóstico de certidumbre $y$ es discutible el cierre obligatorio del Ductus en todos los $\operatorname{cosos}^{12} \cdot 22 \cdot 23$. En los casos presentados fracasó el intento de cierre farmaco. lógico precoz y no se decidió el cierre quirúrgico fundamentalmente por falta de experiencia.

Seguimientos en pacientes con DBP muestra deficiencias pondo-estaturales ${ }^{1}$, rehospitalizaciones por diversas causas hasta en $69 \%$ de los casos en los primeros dos años de vida ${ }^{7}, y$ mayor rjesgo de muerte súbita ${ }^{24}$. Sin embargo el pronóstico global en el largo plazo no es diferente del de otros niños de igual edad gestacional y peso ${ }^{1}$. Aún cuando se requiere un mayor tiempo de seguimiento, en nuestros casos existe una perspectiva favorable referente al futuro de los niños con DBP, considerando la normalidad neurológica liasta la edad en que han sido estudiados. El desarrollo pondo-estatural se vio comprometido sólo en un caso en el seguimiento, pero su tendencia es también hacia la recuperación.

La DBP grave y las complicaciones asociadas a ella comienzan a tener relevancia en nuestro medio. El pronóstico, comparable al de otros recién nacidos de muy bajo peso al nacer, parecen justificar el esfuerzo y el costo del trata. miento, mientras se progresa hacia la meta de su prevención.

\section{RESUMEN}

Se describen los hechos más destácados en cuatro casos de DBP grave, en recién nacidos de 24 a 28 semanas de gestación y de 700 a 1.170 gr. al nacer. Todos fueton tratados con IPPV por períodos entre 41 y 216 días. Entre los trastornos asociados destaca la persistencia del Ductus en 2 ríños, CIV en otro e infecciones por Citomegalovirus en uno. Dos casos se complicaron con obstrucción laríngea severa. La oxigenoterapia fue necesaria entre 98-315 dias, dos pacientes fueron tratados en el domicilio después del alta, por plazos de 27 y 117 dias. El total de días de hospitalización varió entre 100 y 275 días.

El seguimiento, hasta edades corregidas entre 8 y 24 meses, muestra desarrollo psicomotor normal en todos los casos y retardo pondoestatural severo en uno.

El pronóstico de la DBP, comparable al de atros recién nacidos de muy bajo peso, justifica el esfuerzo y el costo del tratamiento.

\section{AGRADECIMIENTOS}

Los autores agradecen a las Enfermeras y Auxiliares del Servicio de Neonatología de la Clínica Alemana, por su dedicación y excelencia en el cuidado de los niños, a los padres por su fe $y$ confianza, $y$ al Dr. Axel Christensen por su asesor ía otorrinolaringológica.

\section{REFERENCIAS}

1. Markestad T., Fitzhardinge, P.M.: Growth and Development in Children recovering from Bronchopulinonary Dysplasia. J. Pediatr. 98:597, 1981

2. Northway, W.H., Rossan, R.C., Porter, D.Y.: Pulmonary diseases following respirator therapy of hyaline membrane disease: bronchopulmonary dysplasia. N. Engl. J. Med. 276: 357, 1967.

3. Auid P.: Oxygen therapy for premature infants. J. Pediatr. 78: 705, 1971

4. Nash. M.A.J.; Bowen, JA.. Langliney, P.C.: Respitutor Lung: A Misnomer. Arch. Pathol. 91: 234. 1971.

5. Banerjee, C.K, Girling, D.J, Wigglesworth, S.S.: Pulmonary fibroplasia in newborn babies treated with oxygen and artificial ventilation. Arc. Dis. Child. 47: 509, 1972.

6. Gairdner, D., Pearson, J,: A growth chart for Pre mature and other Intants. Arch. Dis. Chuld. 46: 783 , 1971.

7. Yu, V.H.L., Orgill, A.A., Lim, S.B. ef al: Growth and development of very low birthweight infants recovering from bronchopulmonary dysplasia. Arch. Dis. Child. 58: 791, 1983.

8. Dudell, G.G., Gersony, W.M.: Patent ductus arteriosus in neonates with severe respiratory disease. $J$. Pediatr. 104: 915,1984

9. Ogden, B.E., Murphy, S., Saunders, G.C. et al.: Lung lavage of newborns with respiratory distress: Prolonged neutrophil influx is associated with Bronchopulmonary Dysplasia. Chest 83: $31 \mathrm{~s}, 1983$.

10. Boynton, B.R., Mannino, F.L, Randel, R.C. et al.: Minimizing bronchopulmonary dysplasia in VLBW infants, J: Pediatr. 104: 963, 1984.

11. Brown, E.R., Stark, A., Sosenko, I. et al.: Bronchopulmonary Dysplasia: possible relationship to pulmonary edema. J. Pediats. 92: 982, 1978.

12. Ehrenkranz, R.A., Ablow, R.C., Warshaw, J.B.: 
Prevention of bronchopulmonary dysplasia with vitamin $\mathrm{E}$ administration during the acute stages of respiratory distress syndrome. J. Pediatr. 95: 873, 1979.

13. Mammel, M.C., Johnson, D.E., Green, T.P. et ol.: Controlled trial of Dexamethasone therapy in infants with bronchopulmonary dysplasia. Lancet 1: $1356,1983$.

14. Donn, S.M., Faix, R.G., Bonagole, R.C.: Dexamethasone for bronchopulmonary dy splasia. Lancet 2 : $460,1983$.

15. Wolfson, M.R., Bhutani, V.X., Schaffer. T.H. et al. Mechanics and energetics of breathing Helium in infants with bronchopulmonary dysplasia. J. Pediatr. 104: 752, 1984.16.

16. Campbell, A.N., Zarfin, Y., Groenveld, M. et al. Low flow oxygen thetapy in infants. Arch. Dis. Child. 58: 795, 1983.

1?. Spitzer, H.R., Fox, W.W., Deliworia-Papadopoulos, $M$.: Maximum diuresis - A factor in predicting recovery from respiratory distress syndrome and the development of bronchopulmonary dysplasia. J. Pediatr. 98: 476, 1981 .

18. Langman, CB., Engle, W.D., Baumgart, $S$. et al. The diuretic phase of respiratory đistress syndrome and its relationship to oxygenation. J. Pediatı. 98 : $462,1981$.

19. Yeh, T.F., Shibli, A., Leu, T. et al: Early Furosemide therapy in premature infants ( $\leqslant 2.000 \mathrm{~g}$ ) with respiratory distress syndrome: A randomized controlled trial. 3 . Pediatr. 105; 603, 1984.

20. Koo, L.C., Warburton, D., Sargent, C.W. et ah. Furosemide acutely decreases airway resistance in chronic bronchopulmonary dysplasia. J. Pediatr. 103: 625, 1983 .

21. Rhodes, P.G, Graves, G.R., Patel, D.M. et al. Minimizing pneumothorax and bronchopulmonary dysplasia in ventilated infants with hyaline membrane disease. J. Pediatr. 103: 634, 1983.

22. Gersony, W.M., Peckham, G.J., Ellison, R, C. et ah: Effects of Indomethacin in premature infants with patent ductus arteriosus. $J$. Pediatr. 102: 895, 1983.

23. Peckham, G.J., Olt, S.M., Ellison, R.C. at at: Clinical course to 1 year of age in premature infants with patent ductus arteriosus: Results of a mulicenter randomized trial of Indomethacin. J. Pediatr. 105: $285,1984$.

24. Werthammer, J., Brown, E.R., Neff, R.K. et al.: Sudden Infant Death Syndrome in infants with Bronchopulmonary Dysplasia. Pediatrics 69: 301, 1982. 